\title{
A case report of an inflammatory myofibroblastic tumor of the neck: A focus on the computed tomography and magnetic resonance imaging findings
}

\author{
KE-JIA CHENG, SHEN-QING WANG and SHUI-HONG ZHOU \\ Department of Otolaryngology, The First Affiliated Hospital, College of Medicine, \\ Zhejiang University, Hangzhou, Zhejiang 310003, P.R. China
}

Received July 26, 2014; Accepted April 9, 2015

DOI: $10.3892 / \mathrm{ol} .2015 .3168$

\begin{abstract}
Inflammatory myofibroblastic tumors (IMTs) of the neck are rare, with only a few patients reported in the literature. The present study discusses the clinical manifestations, radiographic characteristics and management of these tumors, with a focus on imaging modalities. A case of IMT of the neck is presented and the associated literature is reviewed. In total, seven patients in seven English-language studies, including the present case, and one patient in one Chinese-language study were found. On CT scans, all tumors appeared as soft-tissue densities. Upon magnetic resonance imaging (MRI), all tumors displayed a heterogeneous hypointense-isointense signal on T1-weighted sequences and an isointense-hyperintense signal on T2-weighted sequences. All tumors showed enhancement on enhanced CT and MR images. The imaging features of the neck IMTs can be summarized as follows: i) When enhanced, the tumor displays enhancement on CT and MR images; ii) MRI is superior to CT scans in the differential diagnosis of this disease; iii) in general, the lesion displays a hypointense-isointense signal on T1-weighted sequences and an isointense-hyperintense signal on T2-weighted sequences; iv) due to the fibrous tissue in the tumor, delayed enhancement may be observed on gadolinium-enhanced MR images; and v) due to its benign or intermediate features, the tumor is usually a well-defined mass.
\end{abstract}

\section{Introduction}

Inflammatory myofibroblastic tumors (IMTs) are rare soft-tissue neoplasms that have become increasingly prevalent

Correspondence to: Professor Shui-Hong Zhou, Department of Otolaryngology, The First Affiliated Hospital, College of Medicine, Zhejiang University, 79 Qingchun Road, Hangzhou, Zhejiang 310003, P.R. China

E-mail: weiyan127@163.com

Key words: neck, imaging characteristics, inflammatory myofibroblastic tumors, clinical manifestations, surgery in recent years. The tumors are a type of inflammatory pseudotumor (IPT); a mass that contains a mix of myofibroblastic and fibroblastic spindle cells with infiltration of inflammatory cells. IMTs have been referred to as plasma cell granulomas, inflammatory fibrosarcomas and inflammatory myofibrohistiocytic proliferations, reflecting the variable pathological manifestations, and controversial nature and origin (1). An IMT begins as a benign reactive process, and progresses to an intermediate neoplasm with local destruction and recurrence (2)

IMTs can affect people of any age, with a predilection for young adults and children. The tumors occur most commonly in the lung, with the most common extrapulmonary sites as the omentum and mesentery (3). IMTs of the head and neck region are considered to be rare, and can occur in the orbit, maxillary sinus, nasopharynx, parapharyngeal space, larynx, skull base, temporal bone and neck (4-6). IMTs demonstrate various clinical manifestations and pathobiological behaviors, depending on the site affected (3). IMTs in the orbit usually cause only inflammation, which is easily treated by corticosteroids. By contrast, IMTs of the maxillary sinus can recur and can become sarcomatous following incomplete resection. Computed tomography (CT) scans and magnetic resonance imaging (MRI) of IMT lesions differ for each location, and the lesions can be mistaken for other diseases (7). The variable imaging findings of these lesion may be due to the preponderance of spindle cells or inflammatory cells.

In the present study, a rare case of IMT of the neck is described and the associated literature is reviewed. The clinical manifestations, radiographic characteristics and management of these tumors are discussed, with a focus on imaging modalities.

\section{Case report}

A 43-year-old female patient presented to The First Affiliated Hospital (College of Medicine, Zhejiang University, Hangzhou, Zhejiang, China) in April 2012 with a two-month history of a firm, painless, asymptomatic mass in the right side of the neck. No history of trauma, surgery or infection was recorded. Upon physical examination, the patient appeared to be in good health, with no evidence of lymphadenopathy, hepatomegaly or 
splenomegaly. Results of blood tests and a C-reactive protein test were within the normal ranges.

Ultrasonography (USG) examination showed a $3.76-\mathrm{cm}$ mass in the right side of the neck. CT scans revealed a well-defined, non-homogeneous, 3.0x4.2x11.0-cm mass of the right deep neck, with slight enhancement compared with the surrounding tissues. The tumor was close to the cervical vertebrae (from C3 to T2), and had pushed the carotid sheath and thyroid laterally. Bony erosion was not observed in the adjacent osseous structures and the tumor itself was not calcified (Fig. 1). MR images were obtained with T1-weighted, T2-weighted and gadolinium-enhanced T1-weighted sequences. The fusiform, well-defined and non-homogeneous mass displayed a hypointense to isointense signal on T1-weighted sequences and a hyperintense signal on T2-weighted sequences compared with the surrounding tissues. The tumor appeared non-homogeneously enhanced on gadolinium-enhanced T1-weighted images (Fig. 2). The radiographic appearance initially suggested a nerve sheath tumor and the marked enhancement on MRI indicated a possible malignancy.

A USG-guided aspiration biopsy was performed under local anesthesia, and histology showed numerous spindle and inflammatory cells, but the result was non-diagnostic. For surgical excision, a lateral neck incision was made under general anesthesia. A large, firm, reddish-yellow tumor was resected en bloc. Part of the tumor was close to the cervical vertebrae, but bony erosion was not observed, as indicated on CT. Intraoperatively, frozen sections revealed a benign spindle cell tumor. Histologically, the tumor was composed mainly of spindle myofibroblasts with a few infiltrating inflammatory cells. Immunochemistry showed that the tumor cells were positive for smooth muscle actin (SMA), muscle-specific actin (MSA), vimentin, desmin and ALK, but negative for S-100. These results confirmed the diagnosis of a neck IMT. The post-operative recovery was uneventful and 23 months of follow-up revealed no signs of recurrence.

\section{Discussion}

IPT has been used to describe a wide range of reactive and neoplastic lesions, including IMT and certain infectious processes (8). These lesions show similar pathological characteristics, but differ biologically. IMT, a distinctive neoplasm with a few reactive inflammatory cells that occurs primarily in viscera and soft tissues, predominantly affects children and young adults (9). As IMT of the neck is a rare disease, to the best of our knowledge, the present study is the first case report of the lesion. For the present literature review, PubMed was searched regarding IMT of the neck between 1990 and 2013 using the following keywords: 'Inflammatory myofibroblastic tumor' and 'neck'; or 'inflammatory pseudotumor' and neck'; or 'plasma cell granuloma' and 'neck'. Seven patients were found in seven English-language studies, including the present case, and one patient in one Chinese-language study, which described clinical and imaging details (Table I) (7,10-15).

The patients consisted of four males and four females who ranged in age between 12 and 51 years old at initial presentation, with a mean age of 32.25 years old. In the present review, two patients $(25 \%)$ were younger than 18 years, and
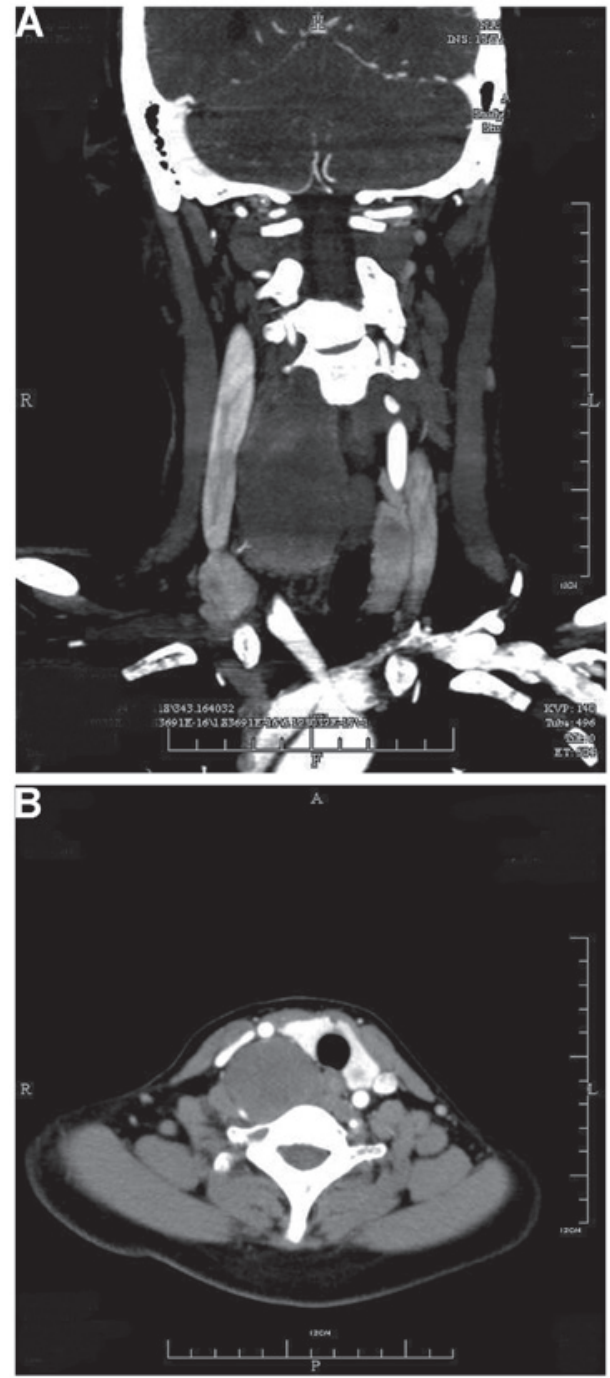

Figure 1. (A) Computed tomography (CT) showing a well-defined, non-homogeneous $3.0 \times 4.2 \times 11.0-\mathrm{cm}$ mass of the right deep neck close to the cervical vertebrae and pushing the carotid sheath laterally. (B) Tumor showing slight enhancement compared with the surrounding tissues on axial CT.

six patients $(75 \%)$ were younger than 40 years. This indicates that IMT of the neck is overrepresented in young people. The most common symptom was a painful or painless neck mass, with four cases affecting the left side, three affecting the right side and one unknown. The etiology of this disease is unknown, as it does not appear to be associated with a history of surgery, infection or trauma. The disease duration ranged between 5 days and 7 years, with the majority recorded as several months. The tumors ranged in size between 30 and $110 \mathrm{~mm}$.

The diagnosis of IMT is based on the histological and immunohistochemical criteria. Microscopically, these lesions are composed of spindle cells and inflammatory cells in a stroma that can be myxoid, fibrotic or hyalinized (16). Mitotic rates are $0-2$ per 10 high-power fields, but abnormal mitotic figures and necrosis are absent. Upon immunohistochemical examination, IMTs usually express antigens indicating myoid differentiation, including SMA, MSA, desmin and vimentin, but are negative for S-100 proteins and epithelial markers. Additionally, $50 \%$ of IMT lesions display ALK protein 


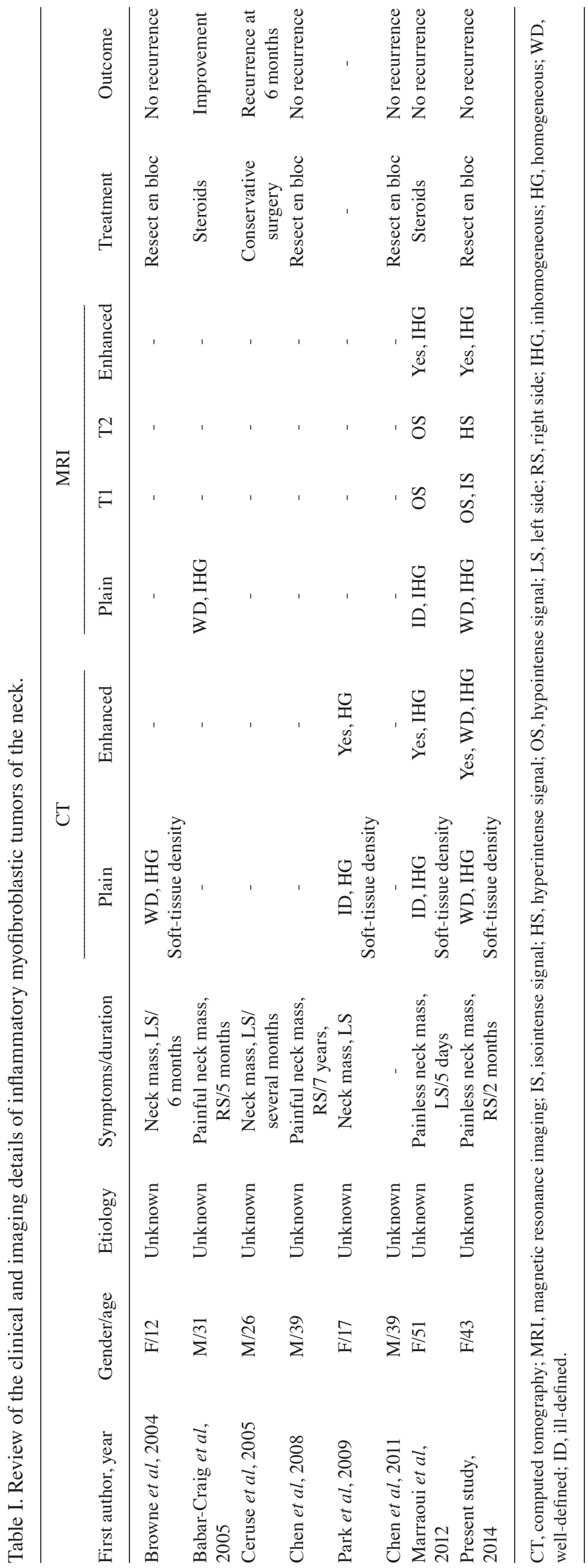



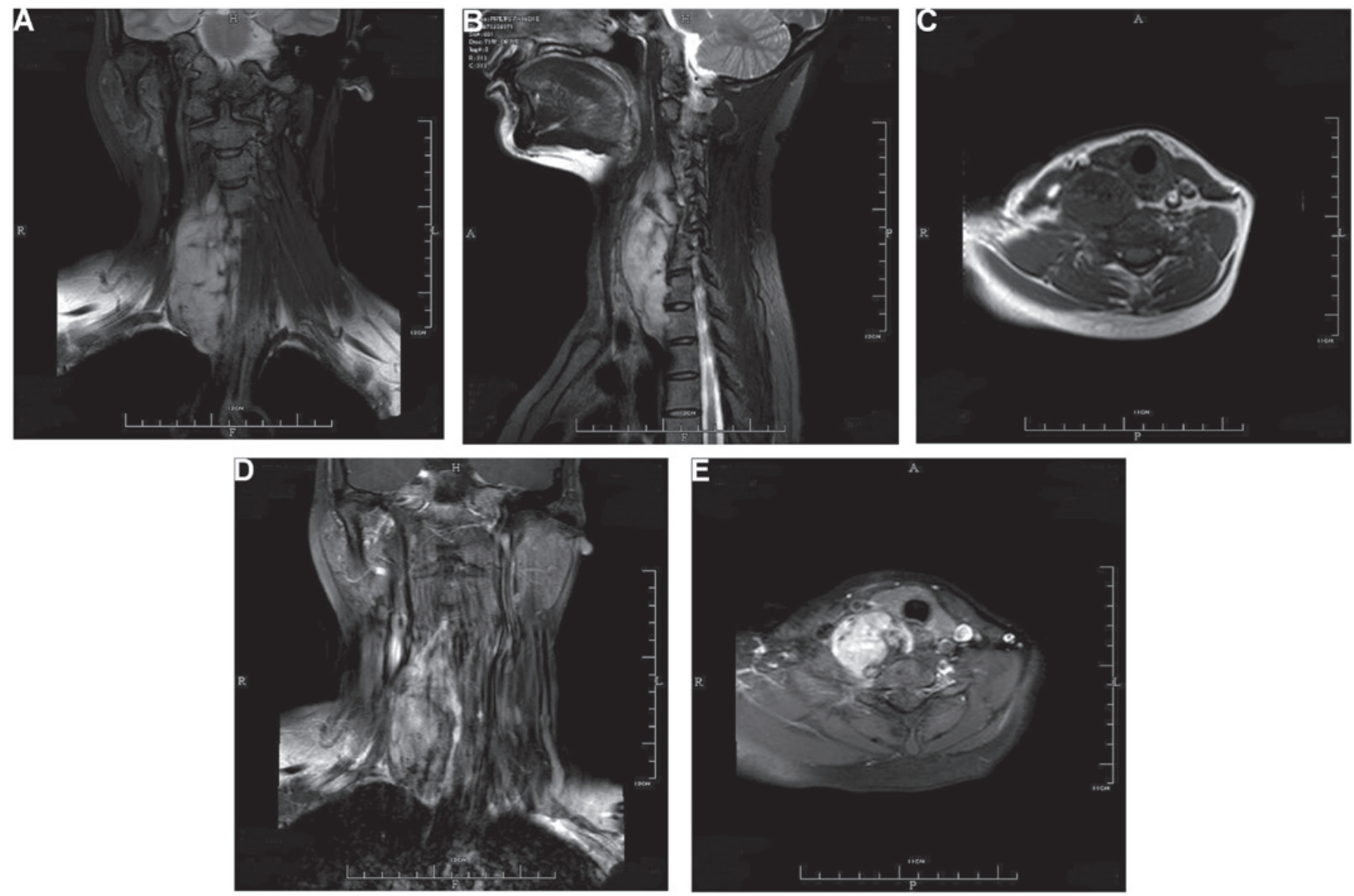

Figure 2. (A) A fusiform, well-defined and non-homogeneous mass displaying a hyperintense signal on coronal T2-weighted magnetic resonance imaging (MRI) compared with the surrounding tissues. (B) The tumor on lateral T2-weighted MRI. (C) The tumor displaying a hypointense to isointense signal on axial T1-weighted MRI. (D) The tumor appearing as a non-homogeneously enhanced mass on coronal gadolinium-enhanced T1-weighted MRI. (E) The tumor on axial gadolinium-enhanced T1-weighted MRI.

overexpression (17). In the present review, three cases included the immunohistochemical findings, with one case positive for SMA, one positive for calponin, and one positive for SMA, MSA, vimentin, desmin and ALK.

The therapy for IMT includes conservative or aggressive surgical resection and steroid treatment. The prognosis for neck IMT is good, although the lesion can recur; however, it rarely metastasizes. In the present review, four lesions were resected en bloc, while two patients opted for steroid treatment; none of these patients experienced recurrence or metastasis. The one lesion that was conservatively resected recurred 6 months later. In our opinion, the gold standard treatment of IMT of the neck is aggressive surgical resection. However, in certain cases of short duration or evident infection, steroids may be preferable.

The differential diagnosis of IMT usually requires immunohistochemical examinations, which require several days to be performed post-operatively. Hence, pre-operative imaging is important to make the correct diagnosis and select options for therapy. IMT can be divided into three main microscopic subtypes: Myxoid-vascular, hypocellular fibrous and compact spindle cell subtypes (18). One previous study concluded that IMTs arising from different locations demonstrate different histological subtypes, which are evident on imaging (19). By contrast, another study found that CT scans of IMTs arising from the lung were not indicative of the pathological characteristics (20).

Kim et al (21) studied the CT features of 10 pulmonary IMTs and found that the tumors all showed mild enhancement, with eight homogeneous and two heterogeneous cases. Takayama et al (22) reported that IMTs of the lung were homogeneous and hypointense on T1-weighted images and hyperintense on T2-weighted images, with delayed enhancement. Yuan et al (23) found that seven IMTs of the maxillary sinus showed heterogeneous enhancement on contrast-enhanced CT and MRI, an isointense signal on T1-weighted images and an isointense to hyperintense signal on T2-weighted images. The MRI findings of IMTs of the liver in further studies were heterogeneous, hypointense or hyperintense on T1-weighted images and isointense or hyperintense on T2-weighted images, with delayed enhancement $(24,25)$. IMT of the limbs exhibited low signal intensity on T1-weighted sequences and intermediate-low signal intensity on T2-weighted sequences on MRI, with enhancement on contrast-enhanced CT and MRI (26).

Due to the rarity of neck IMTs, no review of the imaging characteristics of this disease has been previously reported. In the present review, two cases reported CT and MRI results, two reported CT results only and one reported MRI results only. On the CT scans, all tumors appeared as soft-tissue 
densities. On MRI, all tumors displayed a heterogeneous hypointense-isointense signal on T1-weighted sequences and an isointense-hyperintense signal on T2-weighted sequences. All tumors showed enhancement on enhanced CT and MR images.

The imaging features of neck IMT can be summarized as follows: i) A soft-tissue density, rarely exhibiting calcification or necrosis on CT scans; ii) when enhanced, the mass displays enhancement on CT and MR images; iii) MRI is superior to CT scans in the differential diagnosis of this disease; iv) as this tumor often has multiple components, it usually presents with heterogeneous signals; v) in general, the lesion displays a hypointense-isointense signal on T1-weighted sequences and an isointense-hyperintense signal on T2-weighted sequences; vi) due to the fibrous tissue in the tumor, delayed enhancement may be observed on gadolinium-enhanced MR images; and vii) due to its benign or intermediate features, the tumor is usually a well-defined mass.

\section{Acknowledgements}

The present study was supported by the Zhejiang Province Health Department of Scientific Research Funds (grant no. 2013KYB112).

\section{References}

1. Ma L, Wang K, Liu WK and Zhang YK: Is radical surgery necessary to head and neck inflammatory myofibroblastic tumor (IMT) in children? Childs Nerv Syst 25: 285-291, 2009.

2. Ong HS, Ji T, Zhang CP, Li J, Wang LZ, Li RR, et al: Head and neck inflammatory myofibroblastic tumor (IMT): evaluation of clinicopathologic and prognostic features. Oral Oncol 48: 141-148, 2012.

3. Magill JC, Ferguson MS, Butler CR, Sandison A and Grant WE: Inflammatory myofibroblastic tumour of the tonsil: case report and literature review. J Laryngol Otol 124: 1123-1125, 2010.

4. Lee DH, Shin OR, Cho KJ and Kim JH: Inflammatory pseudotumor in the middle ear cavity. Int J Pediatr Otorhinolaryngol 72: 1569-1572, 2008.

5. Curry JM, King N, O'Reilly RC and Corao D: Inflammatory pseudotumor of the inner ear: are computed tomography changes pathognomonic? Laryngoscope 120: 1252-1255, 2010.

6. Biron VL, Waghray R, Medlicott SA and Bosch JD: Inflammatory pseudotumours of the larynx: Three cases and a review of the literature. J Otolaryngol Head Neck Surg 37: E32-E38, 2008.

7. Chen YF, Zhang WD, Wu MW, Ou-Yang D and Zhang Q: Inflammatory myofibroblastic tumor of the head and neck. Med Oncol 28 (Suppl 1): S349-S353, 2011.

8. Gleason BC and Hornick JL: Inflammatory myofibroblastic tumours: Where are we now? J Clin Pathol 61: 428-437, 2008.

9. Zhang H, Erickson-Johnson M, Wang X, Bahrami A, Medeiros F, Lonzo ML, et al: Malignant high-grade histological transformation of inflammatory myofibroblastic tumour associated with amplification of TPM3-ALK. J Clin Pathol 63: 1040-1041, 2010.
10. Park SB, Lee JH and Weon YC: Imaging findings of head and neck inflammatory pseudotumor. AJR Am J Roentgenol 193: 1180-1186, 2009.

11. Ceruse P, Ramade A, Vautrin R, Crozes C, Dubreuil C and Disant F: Inflammatory pseudotumor of the neck: a long-term result without surgical approach. Otolaryngol Head Neck Surg 132: 812-813, 2005.

12. Babar-Craig H, Gill H, Almeyda R, Wong WL and Farrell R: Inflammatory pseudotumour of the neck with multifocal sites on positron emission tomography scan imaging. J Laryngol Otol 119: 219-221, 2005.

13. Browne M, Abramson LP, Chou PM, Acton R, Holinger LD and Reynolds M: Inflammatory myofibroblastic tumor (inflammatory pseudotumor) of the neck infiltrating the trachea. J Pediatr Surg 39: e1-e4, 2004.

14. Marraoui W, Jean B, Muheish M, Trouillier S, Kemeny JL and Dorcier F: Imaging of inflammatory myofibroblastic cervical tumours: a case report. Diagn Interv Imaging 93: 617-620, 2012.

15. Chen S, Chen H and Qian Z: Inflammatory myofibroblastic tumor of neck: A case report and literature review. Chin J Misdiagn 28: 5551-5553, 2008 (In Chinese).

16. Nonaka D, Birbe R and Rosai J: So-called inflammatory myofibroblastic tumour: a proliferative lesion of fibroblastic reticulum cells? Histopathology 46: 604-613, 2005.

17. Kelleher FC and McDermott R: The emerging pathogenic and therapeutic importance of the anaplastic lymphoma kinase gene. Eur J Cancer 46: 2357-2368, 2010.

18. Coffin CM, Watterson J, Priest JR and Dehner LP: Extrapulmonary inflammatory myofibroblastic tumor (inflammatory pseudotumor). A clinicopathologic and immunohistochemical study of 84 cases. Am J Surg Pathol 19: 859-872, 1995.

19. Horger M, Pfannenberg C, Bitzer M, Wehrmann M and Claussen CD: Synchronous gastrointestinal and musculoskeletal manifestations of different subtypes of inflammatory myofibroblastic tumor: CT, MRI and pathological features. Eur Radiol 15: 1713-1716, 2005.

20. Kakitsubata Y, Theodorou SJ, Theodorou DJ, Nabeshima K, Kakitsubata S and Friedman PJ: Myofibroblastic inflammatory tumor of the lung: CT findings with pathologic correlation. Comput Med Imaging Graph 31: 607-613, 2007.

21. Kim TS, Han J, Kim GY, Lee KS, Kim H and Kim J: Pulmonary inflammatory pseudotumor (inflammatory myofibroblastic tumor): CT features with pathologic correlation. J Comput Assist Tomogr 29: 633-639, 2005.

22. Takayama Y, Yabuuchi H, Matsuo Y, Soeda H, Okafuji T, Kamitani $\mathrm{T}$, et al: Computed tomographic and magnetic resonance features of inflammatory myofibroblastic tumor of the lung in children. Radiat Med 26: 613-617, 2008.

23. Yuan XP, Li CX, Cao Y, Singh S and Zhong R: Inflammatory myofibroblastic tumour of the maxillary sinus: CT and MRI findings. Clin Radiol 67: e53-e57, 2012.

24. Choi BY, Kim WS, Cheon JE, Kim IO, Kim CJ and Yeon KM: Inflammatory myofibroblastic tumour of the liver in a child: CT and MR findings. Pediatr Radiol 33: 30-33, 2003.

25. Yu JS, Park C, Kim JH, Chung JJ and Kim KW: Inflammatory myofibroblastic tumors in the liver: MRI of two immunohistochemically-verified cases. J Magn Reson Imaging 26: 418-421, 2007.

26. Masciocchi C, Lanni G, Conti L, Conchiglia A, Fascetti E, et al: Soft-tissue inflammatory myofibroblastic tumors (IMTs) of the limbs: potential and limits of diagnostic imaging. Skeletal Radiol 41: 643-649, 2012. 